\title{
Physicochemical characterization of cellulose nanowhiskers extracted from oil palm biomass microcrystalline cellulose
}

\begin{abstract}
Cellulose nanowhiskers are lightweight, inexpensive, biocompatible nanomaterials that have found wide range of applications. One of their important applications is in the development of reinforced polymer nanocomposites (PNC). The aim of this study was to isolate cellulose nanowhiskers from oil palm biomass microcrystalline cellulose (MCC) using chemical swelling treatment. Analysis of Fourier transform infrared spectroscopy (FTIR) indicated that chemical swelling did not change the chemical structure of the cellulosic fragments. The morphology of the swelled MCC was observed using scanning electron microscopy (SEM) and the micrographs showed that the aggregated structure of MCC have broken down. The produced cellulose nanowhiskers (CNW-S) were estimated to have less than $20 \mathrm{~nm}$ width and lengths of $300 \mathrm{~nm}$ after treatment, which confirm its nanoscale structure. X-ray diffraction analysis indicated that chemical swelling improve slightly the crystallinity of MCC while maintaining the cellulose I structure. Thermogravimetric analysis (TGA) showed that the $\mathrm{CNW}-\mathrm{S}$ was significantly thermally more stable than MCC, having higher on-set degradation temperature and maximum degradation temperature.
\end{abstract}

Keyword: Cellulose; Nanowhiskers; FTIR; Crystal structure; Thermal properties. 